\title{
La asimilación de experiencias problemáticas a través de narraciones: un estudio de caso
}

\author{
Anna Allepuz-Faus ${ }^{1}$, Isabel Caro ${ }^{2 *}$, Irene Rojo-Serrano ${ }^{1}$ y Ainara Yera-Sabater ${ }^{1}$ \\ 1 Práctica privada \\ 2 Universidad de Valencia. Facultad de Psicologia. Dpto. de Personalidad, Evaluación y Tratamientos Psicológicos
}

\begin{abstract}
Resumen: En este trabajo se busca describir el proceso de asimilación a través de narraciones de una participante, Mónica, que tenía problemas de tipo oncológico y el beneficio de emplear narraciones para dicha asimilación.

Mónica escribió 31 narraciones, durante 8 meses, sobre su experiencia con diversos fibroadenomas y tumores benignos. Estas narraciones se analizaron mediante el modelo de asimilación y la Escala de Asimilación de Experiencias Problemáticas o APES.

El procedimiento de preparar los datos para el análisis con la APES dio como resultado 373 párrafos que fueron codificados con la APES y en relación a ocho temas distintivos y sus correspondientes voces dominantes y no dominantes, relacionados con sus experiencias problemáticas. Los resultados muestran que Mónica alcanzó niveles elevados de asimilación en siete de sus ocho experiencias problemáticas. A pesar de esta asimilación, los datos muestran notables retrocesos, es decir, saltos entre distintos niveles APES.

Palabras clave: Modelo de asimilación; APES; narraciones; investigación de procesos.
\end{abstract}

Title: The assimilation of problematic experiences through narratives: A case study.

Abstract: In this study we aim to describe the assimilation process through our participant narratives, Mónica, who had oncological problems and to explore the advantages of the use of narratives for assimilating these experiences.

Mónica wrote 31 narratives during 8 months about her experiences of having fibroadenomas and benign tumors. These narratives were analized by the assimilation model and the Assimilation of Problematic Experiences Scale or APES.

The procedure to obtain the data ended with a sample of 373 passages that were analized with APES. Those passages were related to eight different topics and their corresponding dominant and non-dominant voices, from their problematic experiences.

Results showed that Mónica reached high levels of assimilation in seven of her eight problematic experiences. Despite this assimilation, data showed remarkable setbacks, in other words, a return from a higher to a lower stage of the APES.

Key words: Assimilation model; APES; narratives; process research.

\section{Introducción}

El presente trabajo se centra en el modelo de asimilación, aunque estudiado con un foco diferente a las investigaciones realizadas hasta la fecha. El modelo de asimilación es de tipo procesual y permite explorar y describir el cambio terapéutico, entendido éste como asimilación de experiencias problemáticas. El modelo desarrollado por el grupo de Stiles (Stiles et al., 1990, 1991) se ha aplicado a una amplia variedad de muestras y tipo de pacientes (Stiles, 2002), e incluso se ha utilizado para estudiar el proceso de aculturación mediante entrevistas (Henry, Stiles \& Biran, 2005). Con el modelo se ha podido explicar el cambio terapéutico en diversos enfoques, como humanistas y experienciales (Honos-Webb, Surko, Stiles \& Greenberg, 1999), psicodinámicos (Knobloch, Endres, Stiles \& Silberschatz, 2001), cognitivos (Caro Gabalda, 2006a, b, 2007, 2008), y cognitivo-comportamentales (Goodridge \& Hardy, 2009; Osatuke, Glick et al., 2005). Aún partiendo de estos estudios, como luego ampliaremos, el presente trabajo no estudia el proceso del cambio a través de sesiones de terapia, sino la asimilación de experiencias problemáticas a través de narraciones. Ofreceremos, por tanto, el proceso de asimilación de diversas experiencias problemáticas de una usuaria en un Servicio de Oncología, tras el diagnóstico de tumores benignos en las mamas. Para ello, emplearemos las narraciones que la participante fue escribiendo durante la duración del estudio. Éste es el primer trabajo de este tipo en esta línea. Por ello, comenzaremos

* Dirección para correspondencia [Correspondence address]: Isabel Caro Gabalda. Universidad de Valencia. Facultad de Psicología. Dpto. de Personalidad, Evaluación y Tratamientos Psicológicos. Avda. Blasco Ibáñez, 21. 46010 Valencia (España). E-mail: isabel.caro@uv.es presentando las ventajas de un estudio narrativo, en este contexto del modelo de asimilación, para pasar después a introducirlo.

Autores como Fisher (1985) o Bruner (1986) han señalado que, los humanos somos, como especie, homo narrans. Con esta premisa, nos parece interesante, como introducción, recoger una idea que sugirió Hardy (1968, p. 5). Para esta autora, “... nosotros soñamos en narrativa, ensoñamos en narrativa, recordamos, anticipamos, deseamos, nos desesperamos, creemos, dudamos, planeamos, revisamos, criticamos, construimos, cotilleamos, aprendemos, odiamos y amamos con la narrativa".

Consideramos, pues, estudiar a nuestra participante desde el enfoque narrativo, porque la narración ofrece la oportunidad de ser útil para la construcción de la experiencia, como medio para crear la identidad (Bruner, 1990) y como vía para dar sentido a nuestra vida (Bell, 2002; Neimeyer, 1994; Ricoeur, 1979; Polkinghorne, 1988; Vogel, 1995).

Sarbin (1986) se refirió a cómo los sujetos elaboran la experiencia a través de la construcción de historias y se relacionan entre sí escuchando las de otros. Esta función de las narraciones es la que queremos subrayar con este estudio. Esto es, el papel que tienen las narraciones como herramienta o recurso para elaborar una experiencia problemática. Las personas narramos lo que nos ocurre. Por tanto, nuestra forma de entender, explicar y comprender lo que vivimos es a través de lo que narramos. Somos, en esencia, seres humanos que utilizamos el lenguaje para expresar lo que vivimos. Como Ricoeur (1997) afirmó, la forma discursiva más apropiada para describir la acción humana es la narrativa. Las narraciones se preocupan de cómo los humanos tratan de llegar a una solución, una clarificación o "desenredar /desentrañar" una situación incompleta. En la misma línea, 
Hewitt (1989) explica que, “... la mayor parte de la conversación mediante la que nosotros construimos y reconstruimos (nuestras biografías) ocurre dentro de nosotros y no en interacción con otros" (p. 183). Esta frase define bastante bien el valor que nosotras le damos a la función tan potente que tiene la escritura narrativa como vía para crear nuestra identidad y nuestra historia de vida.

Autores como Frank (1992, 1994, 1995, 1998, 2000) y Bell (2002), entre otros, le han dedicado una especial atención al fenómeno de escribir sobre la enfermedad y los beneficios que se derivan de ello, aspecto fundamental del presente estudio. Estos beneficios de la escritura terapéutica incluyen mejorías en la salud, el bienestar psicológico, el funcionamientos fisiológico y el funcionamiento general (Kerner \& Fitzpatrick, 2007). En esta línea, Pennebaker y sus colegas postularon que "escribir es beneficioso en el grado en que la gente está dispuesta a expresar sus emociones en palabras" (Pennebaker, Mayne \& Francis, 1997, p. 864). Otros estudios han demostrado que escribir sobre acontecimientos traumáticos puede promover un afrontamiento efectivo (Lumley \& Provenzano, 2003; Pennebaker, Colder, \& Sharp, 1990), disminuir síntomas depresivos por atenuar los efectos emocionales negativos de los pensamientos intrusivos (Lepore, 1997) y disminuir la ocurrencia de enfermedad física debido al estrés (Greenberg, Wortman, \& Stone, 1996; Pennebaker, Kiecolt-Glaser, \& Glaser, 1988).

La narración influye, como Frank (1995) consideró, en la reevaluación de la identidad. En este sentido, esta reevaluación, según recoge este autor, es el tema central de las narrativas de enfermedad. Argumenta que el centro de cualquier narrativa de la enfermedad es una epifanía, después de la cual la persona reevalúa su lugar en el mundo. Frank (op.cit.) explica que durante la enfermedad, o después de ella, no solamente el cuerpo está amenazado sino que la identidad también. Así, consideramos a la narrativa teniendo una función terapéutica centrada en la reconstrucción de la identidad.

Puesto que el modelo de asimilación se centra en el funcionamiento del sí mismo, creemos que es factible relacionar y estudiar la asimilación de experiencias problemáticas desde una perspectiva narrativa. Esta relación ya se ha planteado inicialmente en el contexto del modelo de asimilación (Osatuke et al., 2004; Stiles, Honos-Webb \& Lani, 1999). Aunque hay que señalar que el origen del modelo se emplaza en los estudios sobre esquemas de Piaget $(1962,1970)$, en el modelo de Rogers (1959) sobre el desarrollo del sí mismo, y en el trabajo de Elliott (1985) sobre momentos terapéuticos significativos. En la actualidad, los principales estudios se basan sobre el concepto de vo\%:

Este concepto se deriva de las teorías sobre el sí mismo desde una perspectiva dialogal (Bajtin, 1929/1984; Hermans, 1996; Hermans \& Dimaggio, 2004; Hermans, Kempen \& van Loon, 1992; Dimaggio \& Stiles, 2007). Desde esta perspectiva se considera que el sí mismo se caracteriza por ser una comunidad de voces, interconectadas entre sí por el diálogo. Así, podríamos señalar dos tipos de voces. Las voces do- minantes (VD) corresponderían a nuestros recursos habituales, a nuestra forma habitual de pensar, sentir y comportarnos. Estos recursos surgen en función del contexto. Por ejemplo, nuestros recursos como terapeutas no son los mismos que nuestros recursos para conducir y según la situación en la que estemos emplearemos unos y no otros.

Por regla general, a medida que vamos teniendo experiencias éstas se asimilan en nuestro sí mismo, lo enriquecen, y se convierten en recursos útiles. Sin embargo, a veces, tenemos experiencias que no podemos asimilar, que nos son extrañas, o problemáticas, ya que "chocan" con nuestra comunidad usual de voces. Desde esta perspectiva, estas experiencias se consideran problemáticas reflejando toda una serie de voces no dominantes (VND). Por ejemplo, si una paciente con problemas de ansiedad tiene como voz dominante: "necesito sentir que controlo", cuando tenga experiencias tales como: ansiedad, palpitaciones, o cualquier tipo de síntoma (voces no dominantes) que no pueda controlar, se sentirá mal, más ansiosa y con una mayor exigencia de controlar dichas sensaciones de ansiedad. Estas experiencias o voces no dominantes se rechazarán y a menos que el paciente logre articular un diálogo entre su parte dominante y su parte no dominante, la experiencia será difícil de asimilar (Honos-Webb \& Stiles, 1998).

Este diálogo comienza desde el mismo momento en que la atención del paciente se centra en la experiencia problemática y va tomando, por tanto, formas diversas (Stiles, 2011). Ahora bien, para que se dé una asimilación es necesario que se establezca un puente de significado entre las voces. Este puente de significado se puede entender "como una expresión o caracterización de la experiencia problemática de forma que ésta es aceptable y comprensible tanto para la voz problemática como para la dominante" (Stiles \& Glick Brinegar, 2007, pág. 103).

Este diálogo entre voces, este juego entre lo dominante y lo no dominante, se articula mediante la APES o Escala de Asimilación de Experiencias Problemáticas (Stiles, et al., 1991). Cada uno de los niveles APES refleja el rechazo, la aparición, la comprensión (en donde se apreciaría de forma más clara el puente de significado) o la acción sobre estas voces dominantes y experiencias problemáticas y el proceso a seguir cara a su asimilación.

Los 8 niveles de la APES, han sido descritos en Stiles et al., (1991) y en trabajos posteriores (i.e., Honos-Webb \& Stiles, 1998; o Stiles, 2002). En el Cuadro 1 tenemos una descripción de la Escala APES. Los niveles APES se explican o se ofrecen de manera lingüística. Por ejemplo, se puede decir que una experiencia está en un nivel de Clarificación. Pero también se puede manifestar el nivel APES de manera numérica, a saber, diciendo que la experiencia está en un nivel 3 (o Clarificación). La estimación numérica ofrece una estimación más precisa del nivel APES en el que se encuentra el paciente y permite, igualmente, ofrecer niveles intermedios (así 2.5 marcaría una puntuación a medio camino entre Surgimiento y Clarificación). 
Cuadro 1. Niveles en el proceso de asimilación (a partir de Stiles, Honos-Webb y Lani, 1999).

\begin{tabular}{|c|c|}
\hline Nivel & Descripción \\
\hline 0.- Supresión/Disociación & $\begin{array}{l}\text { El contenido no está formado; el paciente no se da cuenta del problema. El cliente/paciente no trabaja en el } \\
\text { problema. La experiencia se considera evitada o desviada si hay evidencia de evitar activamente temas emo- } \\
\text { cionalmente molestos (i.e., cambiar de tema). }\end{array}$ \\
\hline $\begin{array}{l}\text { 1.-Pensamientos no desea- } \\
\text { dos/Evitación }\end{array}$ & $\begin{array}{l}\text { El contenido refleja el surgimiento de pensamientos asociados con el malestar. El paciente prefiere no pen- } \\
\text { sar sobre ello; los temas los propone el terapeuta o las circunstancias externas. El terapeuta pregunta algo y } \\
\text { el paciente cambia de tema, algo así como "tengo que ser duro y no pensar en esto". El afecto es más des- } \\
\text { tacado que el contenido e implica fuertes sentimientos negativos -ansiedad, miedo, cólera, tristeza. }\end{array}$ \\
\hline $\begin{array}{l}\text { 2.-Reconocimiento va- } \\
\text { go/Surgimiento }\end{array}$ & $\begin{array}{l}\text { El paciente se da cuenta de la existencia de una experiencia problemática, y describe pensamientos poco } \\
\text { agradables asociados con los pensamientos, pero no puede formular el problema con claridad. Sabe lo que le } \\
\text { molesta. El afecto incluye dolor psicológico agudo o pánico asociados con los pensamientos problemáticos y } \\
\text { las experiencias. }\end{array}$ \\
\hline $\begin{array}{l}\text { 3.-Enunciar el proble- } \\
\text { ma/Clarificación }\end{array}$ & $\begin{array}{l}\text { Ves lo que pasa y lo que lo impide. El contenido incluye un enunciado claro del problema -algo en lo que se } \\
\text { puede trabajar. El paciente enuncia el problema pero no hace ninguna conexión sobre la causa del proble- } \\
\text { ma. El afecto es negativo pero manejable, no hay pánico. }\end{array}$ \\
\hline 4.-Comprensión/Insight & $\begin{array}{l}\text { La experiencia problemática se coloca en un esquema, es formulada y comprendida con claros vínculos co- } \\
\text { nectivos. El afecto puede ser variado, con algunos reconocimientos desagradables, pero con curiosidad o } \\
\text { incluso con sorpresa del tipo del "ajá". Niveles del } 4.1 \text { al } 4.9 \text { reflejan progresivamente mayor claridad o ge- } \\
\text { neralización de la comprensión, asociada, por lo general con un afecto positivo (o negativo disminuyendo) }\end{array}$ \\
\hline 5.-Aplicación/Elaboración & $\begin{array}{l}\text { La comprensión se emplea para trabajar en el problema; hay una referencia a esfuerzos específicos para re- } \\
\text { solver el problema, aunque sin un éxito completo. El paciente puede describir que está considerando alter- } \\
\text { nativas o seleccionando, sistemáticamente, cursos de acción. }\end{array}$ \\
\hline 6.-Solución del problema & $\begin{array}{l}\text { El paciente logra una solución a un problema específico. El afecto es positivo, satisfecho, orgulloso del lo- } \\
\text { gro. Niveles del } 6.1 \text { al } 6.9 \text { reflejan generalizar la solución a otros problemas y construir las soluciones sobre } \\
\text { patrones usuales o habituales de conducta. El cliente/paciente ha practicado la solución fuera de la consul- } \\
\text { ta. }\end{array}$ \\
\hline 7.- Dominio & $\begin{array}{l}\text { El paciente emplea, con éxito, soluciones en nuevas situaciones; } \\
\text { Esta generalización es bastante automática, no destacada. El afecto es positivo cuando se habla del tema, } \\
\text { pero neutro (i.e. esto no es ya un problema o algo por lo que preocuparse). El problema ha dejado de ser } \\
\text { un problema. }\end{array}$ \\
\hline
\end{tabular}

Gracias a la escala APES se ha podido realizar la serie de estudios que reseñábamos al inicio y que nos permiten, entre otras cosas, asumir, en primer lugar, una relación entre asimilación y cambio. En este sentido, pacientes con cambios pretest-postest (en medidas estándar) manifestaban niveles más elevados de asimilación, por regla general, superiores al nivel 4 o Insight (Caro Gabalda, 2006a; Detert, Llewelyn, Hardy, Barkham \& Stiles, 2006; Stiles, 2002). Por el contrario, pacientes con un escaso cambio pretest-postest no presentaban niveles altos de asimilación, por regla general inferiores a 3 o Clarificación (Caro Gabalda, 2006b; Detert et al., 2006).

Por otro lado, se ha encontrado un patrón de asimilación diferente en función del tipo de terapia estudiada. Así terapias de corte humanista presentan una secuencia de asimilación (medida a través de la APES) bastante regular, con escasos saltos entre niveles APES (véase Honos-Webb, Stiles, Greenberg \& Goldman, 1998; Osatuke, Glick et al., 2005). Mientras que cuando la terapia estudiada ha sido de tipo cognitivo la secuencia de asimilación se caracteriza por avances y retrocesos entre los niveles APES (Caro Gabalda, 2006a, 2007, 2008; Goodridge \& Hardy, 2009; Osatuke, Glick et al., 2005). Es decir, el paciente avanza a lo largo del proceso de asimilación, pero en determinados momentos retrocede a niveles inferiores de asimilación. Dentro del mode- lo se considera un retroceso, un salto atrás en las mediciones obtenidas con la escala, superior a 1. No obstante, a pesar de estos retrocesos se puede constatar en los pacientes un cambio terapéutico medido con cuestionarios estándar (Caro Gabalda \& Stiles, 2009; remitido para su publicación; Goodridge \& Hardy, 2009; Osatuke, Glick et al., 2005).

Se han presentado diversas hipótesis sobre el por qué de estos retrocesos, revisadas inicialmente por Stiles (2005). Estudios recientes explican dichos retrocesos en un contexto terapéutico de tipo cognitivo mediante dos explicaciones principales que suponen, ambas, el juego y la dinámica de interacción paciente-terapeuta, así como la multiplicidad de voces en el sí mismo de los pacientes (Caro Gabalda \& Stiles, remitido para su publicación).

La primera explicación, la zona de desarrollo próximo, basada sobre el concepto del mismo nombre de Vygotski (Leiman \& Stiles, 2001), asume que el retroceso se da cuando terapeuta y paciente están en un nivel APES diferente, que no comparten. O por decirlo de otra manera, que el terapeuta guía o fuerza demasiado al paciente y éste, no le sigue. Por ejemplo, pedirle al paciente una conclusión no distorsionada y que el paciente sea incapaz de ofrecer una. Desde el punto de vista del modelo de asimilación dicha situación podría implicar un retroceso medido con la APES. 
Por otro lado, se ha visto que en numerosas ocasiones, el terapeuta guía al paciente para que éste se centre en determinados aspectos como, por ejemplo, en pensamientos negativos en un contexto cognitivo. Si el paciente le sigue y logra explorar y focalizarse en este material (afecto y pensamientos negativos) el paciente podría manifestar un retroceso medido con la APES. Esta actividad terapéutica, facilita que el paciente balancee su vieja forma de pensar con otra diferente, alternativa (bipótesis de la balanza) ya que, en un contexto cognitivo, las conclusiones alternativas siempre deben darse ante pensamientos negativos y es importante que el paciente se focalice en ellos para modificarlos.

En función de lo anterior consideramos, pues, importante, estudiar por primera vez a una participante que no recibe terapia, sino que escribe sobre su experiencia problemática. Así, los objetivos de este estudio son dobles e interrelacionados. Por una parte nos proponemos describir el proceso de asimilación de nuestra participante a través de narraciones. Y por otra, explorar las ventajas en sí del uso de narraciones para asimilar una experiencia problemática de tipo oncológico.

\section{Método}

\section{Descripción de la participante}

Nuestra participante es una mujer soltera de 26 años que dio su consentimiento informado para este estudio. Mónica (un pseudónimo) estudiaba $5^{\circ}$ curso de licenciatura en una Universidad española en el momento de su participación.

Inicialmente el caso fue seleccionado tras una entrevista con nuestra participante en donde explicó a una de las firmantes de este estudio que estaba inmersa en un proceso oncológico (véase apartado de Procedimiento). Ella se refería a sus tumores continuamente y sobre cómo le afectaban. A pesar de que su diagnóstico no fuera de cáncer, sino de fibroadenomas, lo vivía como si se sintiera amenazada por un cáncer. Seleccionamos, pues, a Mónica porque tenía una experiencia problemática que vivía de manera angustiosa.

A principios de mayo de 2009 se le plantea entrar en el estudio en donde se trataría de escribir semanalmente con el fin de plasmar cómo se siente, qué piensa y qué hace respecto a sus tumores. Al aceptar, firma un consentimiento informado redactado para el estudio en concreto y se dejan claras las condiciones para ello. Mónica escribió treinta y una narraciones (una semanal) a lo largo de ocho meses, desde mayo de 2009 hasta principios de enero de 2010.

\section{Historia médica}

En junio de 2008 se le detectan tres tumores en la mama. En noviembre del mismo año, es intervenida quirúrgicamente en el Servicio de Oncología del Hospital de su ciudad para extraer un tumor (tumorectomía) en la mama derecha. Exactamente le extirparon el tumor y parte de la glándula mamaria.
A los pocos meses, Mónica se notó (como ella afirma en su primera narración) otros bultos en los ovarios, y se le comunicó que tenía en cada ovario una neoplasia. En marzo de 2009 se le remite al Instituto Valenciano de Oncología (IVO). Aparecen varias neoplasias ginecológicas y aparece otro tumor en la misma mama que había sufrido la tumorectomía. Tiene cita cada mes en el Instituto de Oncología y se exploran y controlan periódicamente sus fibroadenomas hasta la finalización del estudio.

En la primera narración relata que los niveles de malignidad de los tumores aumentan, por lo que cada mes, y tras cada visita, las alternativas terapéuticas se reducen cada vez más a la radioterapia en caso de que ésta fuera necesaria. El tratamiento que lleva son corticoides y progesterona con el fin de mitigar el dolor en el pecho. En la actualidad sólo requiere asistir a revisiones periódicas.

\section{Evaluación psicológica}

Queremos comentar en este punto, que a pesar de que el postest fue respondido en diciembre de 2009, Mónica escribió la última narración a posteriori. El estado psicológico se evaluó a través del HAD (Hospital Anxiety and Depression Scale) (Zigmond \& Snaith, 1983), el Mini-Mac (Mini Escala de Ajuste Mental al Cáncer) (Watson et al., 1994) y El Cuestionario de 90 Síntomas de Derogatis (en adelante, SCL-R 90) en su adaptación española (Derogatis, 2002). Mónica contestó, además, a una Entrevista Semiestructurada que fue útil para la conceptualización de las voces.

En la Tabla 1 tenemos las puntuaciones de Mónica en el pretest (mayo de 2009) y en el postest (diciembre de 2009) en las pruebas ya mencionadas.

Como vemos hay una disminución en el postest en la subescala de Ansiedad en al HAD, aunque sigue estando en un nivel alto, según los intervalos establecidos (Caro Gabalda e Ibáñez Guerra, 1992; López-Roig et al., 2000). En cuanto a la subescala de Depresión manifiesta una puntuación intermedia.

Respecto al Mini-Mac sube en Espíritu de Lucha y baja en Desamparo y Preocupación Ansiosa, aunque en ambas se mantiene por encima de la media. Sube ligeramente en Fatalismo, pero se mantiene por debajo de la media, y baja ligeramente en Evitación Cognitiva, aunque se mantiene por encima de la media.

En cuanto al SCL-90-R y siguiendo las normas de Derogatis (2002) se consideraría que Mónica estaría en riesgo en Ansiedad, Hostilidad, y GSI. Este es un indicador directo del nivel de gravedad de los síntomas. Y las escalas en las que consideraríamos, según los baremos, que habría presencia de patología severa serían: Depresión y Psicoticismo. Con ello podemos decir que Mónica presenta sentimientos de alineación social. Siguiendo con la interpretación de las puntuaciones, vemos que existe una alta intensidad de los síntomas que presenta en esta prueba, ya que la puntuación obtenida en el PSDI fue de 85 (puntuación centil). Finalmente, se produce una disminución en el postest en todas las subescalas salvo en Depresión. 
Tabla 1. Puntuaciones de Mónica en diversas pruebas clínicas en el Pretest y el Postest.

\begin{tabular}{llc}
\hline & PRETEST (Mayo 2009) & POSTEST (Diciembre 2009) \\
\hline HAD-A (Ansiedad) & 16 & 10 \\
HAD-D (Depresión) & 9 & 9 \\
Espíritu de lucha & 9 & 10 \\
Desamparo & 11 & 9 \\
Preocupación ansiosa & 30 & 24 \\
Evitación cognitiva & 11 & 12 \\
Somatizaciones & 11 & 10 \\
Obsesiones y compulsiones & 50 & 25 \\
Sensibilidad interpersonal & 60 & 40 \\
Depresión & 25 & 30 \\
Ansiedad & 80 & $80-85$ \\
Hostilidad & 75 & $20-25$ \\
Fobias & 75 & 30 \\
Ideación paranoide & 5 & 5 \\
Psicoticismo & 15 & 5 \\
Indice global de severidad (GSI) & 90 & 20 \\
Total de síntomas positivos (PST) & 75 & 40 \\
Indice positivo de malestar (PSDI) & 40 & 40 \\
\hline
\end{tabular}

Nota: HAD (Puntuaciones directas): Escala Hospitalaria de Depresión y Ansiedad (Zigmond \& Snaith, 1983). MINI-MAC (Puntuaciones directas): Mini Escala de Ajuste Mental al Cáncer (Watson et al.,, 1994). SCL-90-R (Puntuaciones centiles): Cuestionario de 90 síntomas de Derogatis (2002).

\section{Procedimiento}

La muestra para el presente estudio la proporcionó Mónica, gracias a su colaboración durante 8 meses. Durante este tiempo escribió semanalmente narraciones acerca de su experiencia oncológica que enviaba por correo electrónico.

Para preparar los datos con el fin de crear un catálogo para el análisis de la asimilación se sigue una serie de pasos establecidos por el grupo de Stiles, publicados en diversos trabajos (Stiles et al., 1990; Stiles, Meshot, Anderson \& Sloan, 1992; Honos-Webb, Surko \& Stiles, 1998; HonosWebb, et al., 1999; Knobloch, Endres, Stiles \& Silberschatz, 2001; Lani et al., 2002). La idea básica es identificar experiencias problemáticas (o VND), junto con las VD y observar cómo cambian. El material con el que hemos contado son las 31 narraciones escritas semanalmente, analizadas por completo.

Para obtener la muestra de esta investigación hemos seguido los pasos que detallaremos a continuación.

Familiarización. Este punto pretende que el investigador llegue a estar totalmente familiarizado con los datos a manejar, con el caso que va a estudiarse.

Identificar y elegir los temas. En este punto se ramifican las experiencias problemáticas, aislando las voces, y concretando cada tema. También se le llama a este paso: catalogar, es decir, ir seleccionando los párrafos relevantes buscando la actitud que expresa un paciente hacia un objeto. Una actitud/posición es una creencia, sentimiento, evaluación o una acción. El objeto sería, una persona, cosa objeto, acontecimiento o situación hacia la cual se expresa esa actitud o acción. Por ejemplo sentir ansiedad (actitud) en las relaciones sociales (objeto).
Para identificar las voces se debe prestar atención a los Insight (nivel 4 APES). Esto significa encontrar la asimilación cognitiva de una experiencia problemática en un esquema o voz activos. De manera que esto garantiza que se analizan experiencias sobre las que se ha producido algún cambio. Además, las voces dominantes y no dominantes se buscan en las narraciones atendiendo al contenido, a la intención comunicativa, al afecto que demuestran, etc. (Osatuke et al., 2004). Para identificarlas fue útil el pase a Mónica, en el pretest, de una Entrevista Semiestructurada.

Para hacer la selección del material se busca del catálogo disponible para cada tema los párrafos donde se habla de él. Observamos cuál es la actitud hacia un objeto y esto nos sirve como párrafo a analizar. Hecho todo lo anterior se dispone de una muestra con párrafos significativos de cada tema donde aparecen las voces dominantes y no dominantes. Tras ello se aplica la APES.

Para aplicar la APES se siguió los dos manuales desarrollados por el grupo de Stiles (Honos-Webb, Surko \& Stiles, 1998; Lani, et al., 2002). Para ello fue muy útil el empleo de marcadores o patrones en el discurso terapéutico que son fáciles de reconocer y que están, empírica y teóricamente, vinculados con estas fases (Honos-Webb, et al., 1999).

Empleo de jueces. Aunque los datos cualitativos dependen mucho del contexto y del conocimiento del caso, es aconsejable buscar un acuerdo entre jueces. Con este fin, las autoras de este trabajo, revisaron y acordaron, en primer lugar, los párrafos seleccionados, así como las principales experiencias y voces manifestadas por Mónica en sus narraciones. Acordado todo ello, y también mediante consenso (codificación y acuerdo en cada código) se aplicó la APES a los párrafos seleccionados. Cualquier duda o problema surgido se 
resolvió por consenso entre las jueces en la línea de trabajos del grupo de Stiles (Schielke et al., 2009) en una serie intensiva de reuniones y revisiones a lo largo de seis semanas.

\section{Muestra}

Este procedimiento dio como resultado una muestra de 373 párrafos, extraída de sus 31 narraciones, que hacen referencia a 8 temas distintos que fueron codificados con la APES, siguiendo el procedimiento de acuerdo entre jueces ya mencionado. La muestra aparece descrita en la Tabla 2.

Tabla 2. Muestra: Temas, $N$ de párrafos y voces de Mónica.

\begin{tabular}{|c|c|c|}
\hline Temas & & Voces \\
\hline $\begin{array}{l}\text { Tema1 Temor/incertidumbre/ miedos sobre la enferme- } \\
\text { dad }\end{array}$ & $n=144$ & $\begin{array}{l}\text { VND : "siento miedo, incertidumbre, temor" } \\
\text { VD: "debería sentirme tranquila, sosegada, sin tensión" }\end{array}$ \\
\hline Tema2 Afrontamiento del problema & $n=118$ & $\begin{array}{l}\text { VND: "No sé cómo afrontar el problema" } \\
\text { VD: "Yo sé afrontar los problemas de mi vida". }\end{array}$ \\
\hline Tema 3 Control emocional/ inestabilidad emocional & $n=41$ & $\begin{array}{l}\text { VND: "Me cuesta controlar/gestionar mis emociones" } \\
\text { VD: "Yo controlo mis emociones" }\end{array}$ \\
\hline Tema 4 Derecho a quejarse & $n=22$ & $\begin{array}{l}\text { VND: "Necesito quejarme" } \\
\text { VD: "No debo quejarme." }\end{array}$ \\
\hline Tema 5 Evitar el sufrimiento a los demás & $n=19$ & $\begin{array}{l}\text { VND: "Ver a mis seres queridos sufrir" } \\
\text { VD: "Yo no tendría que hacerles sufrir" }\end{array}$ \\
\hline Tema 6 Culpabilidad & $n=12$ & $\begin{array}{l}\text { VND: "Me siento culpable por no visitar a mis familiares enfermos" } \\
\text { VD: "Debería visitar a mis familiares enfermos" }\end{array}$ \\
\hline Tema 7 Seguridad en mi mundo & $n=9$ & $\begin{array}{l}\text { VND: "Percibo mi mundo como inseguro" } \\
\text { VD: "Mi mundo es seguro" }\end{array}$ \\
\hline Tema 8 Ordenar el exterior para ordenar el interior & $n=8$ & $\begin{array}{l}\text { VND: "Siento la vida desordenada" } \\
\text { VD: "Yo tengo en orden mi vida" }\end{array}$ \\
\hline & $N=373$ & \\
\hline
\end{tabular}

Una de las consecuencias de este procedimiento es la de permitirnos describir al participante desde el modelo de asimilación. Un ejemplo resumido de ello nos aparece en la Figura 1.

\section{Resultados}

A continuación presentaremos los resultados más relevantes encontrados en esta investigación. En las Figuras 2 y 3 nos aparece el proceso de asimilación de Mónica en los principales temas analizados. Por cuestiones de espacio no podemos ofrecerlas todas. El patrón que muestran estas dos figuras es semejante al patrón de las restantes experiencias problemáticas. Es decir, niveles elevados de asimilación (5: Aplicación y 6: Solución del Problema) en determinados momentos y retrocesos en el proceso de asimilación. La única experiencia en la que Mónica no alcanza un nivel mayor a 3 en los 8 párrafos en los que apareció en las transcripciones, fue la de "organizar el exterior para organizar el interior" (véase Tabla 2). A continuación, ofreceremos una breve descripción cualitativa del proceso de asimilación en uno de sus temas principales: "Incertidumbre, miedo y temor sobre el cáncer" por ser ésta la experiencia que alcanza niveles más elevados de asimilación y por ser la que aparece, con mayor frecuencia, en sus narraciones (véase Figura 2).
Proceso de asimilación de la experiencia problemática del "temor/ incertidumbre/ miedos sobre la enfermedad"

Las voces correspondientes a este tema son: Mónica la que siente miedo, incertidumbre y temor como VND y Mónica la tranquila, sosegada, sin tensión como VD.

El tema ya aparece en la primera narración con una cadena de muchas quejas psicológicas (correspondiente al nivel 2 de la APES), en forma de emociones dolorosas cuando explica el momento del diagnóstico de los fibroadenomas. Mónica escribe: "[(VND) Lloré, me lamenté, confesé mi miedo, mis dudas iy si me pasa algo? ¿Y si no sale bien? (2. Surgimiento. Marcador: Sentirse vulnerable)]. [Aun hoy me planteo estas preguntas que siguen generando una sensación de desespero y rabia" (3. Clarificación. Marcador: Lo que pienso (o he hecho) influye en el problema)]. Tras ello, Mónica une y clarifica el hecho de sentirse con miedo cuando se plantea lo que le puede ocurrir (nivel 3 APES).

En la segunda narración, al comentar que de nuevo debe acudir a hacerse una ecografía, vuelven a aparecer emociones dolorosas y una necesidad de cambio. Tras expresar el afecto negativo, realiza una Clarificación situándose casi a las puertas del Insight, por lo que se codificó con un 3.5. Mónica escribió: "Veamos, ... (VND) tras la noticia, empiezas a reflexionar y lo haces siempre partiendo de la incertidumbre, con lo cual saco conjeturas que me limitan, me incapacitan en mi dia a dia, que me mantienen apática, sin ganas de hacer, y lo peor a mi juicio, sin ganas de sentir" (3.5. Clarificación. Marcador: Lo que pienso (o he hecho) influye en el problema)]. Una visita al médico en el que se le detectan signos de malignidad en uno de los tumores 
coincide con la aparición (en la narración \#4) de emociones dolorosas que corresponderían a niveles 2 de la APES.

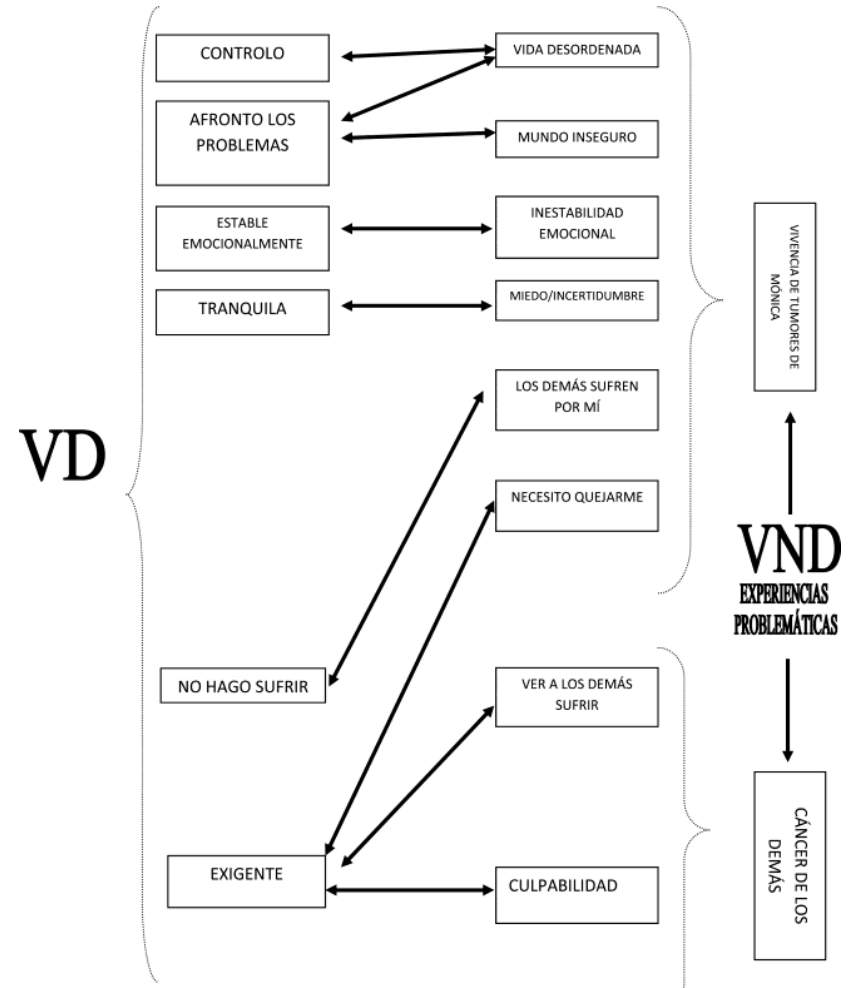

Figura 1. Voces dominantes y voces no dominantes de Mónica

Es a partir de la narración \#6, cuando Mónica empieza a alcanzar niveles más altos de la APES, es decir 5 (Aplicación) y 6 (Solución del Problema). Por ejemplo, Mónica escribió en la narración \#6: "[CVD) Me sentía más tranquila de lo habitual, pues me podía imaginar los resultados, (5. Aplicación. Marcador: Bastante, pero no todavía resolviendo el proble$\mathrm{ma}$ dado que una semana antes habia tenido una visita en el IVO, y ya me habian informado del estado de los tumores, y su evolución. Asi que a diferencia de otras ocasiones, [(VD) estaba más tranquila y sosegada, no sentía tanta ansiedad, y mi cuerpo lo notaba sin la tensión de otras veces" (5. Aplicación. Marcador: Bastante, pero no todavía resolviendo el problema)].

En la narración \#7 también aparecen estos niveles, por ejemplo: "En cierto modo, [(VD) creo que estoy acostumbrándome y habituándome a la situación presente; a la espera" (5. Aplicación. Marcador: Bastante, pero no todavia resolviendo el problema)7

Sin embargo, en la narración \#9 escribió sobre algo importante. Mónica tenía una ecografía en el hospital de su ciudad. Ante este hecho, ella escribió que parecía estar acostumbrada a la situación (nivel 6, Solución del Problema). Sin embargo, explica, que el diagnóstico de los doctores de su hospital y el del IVO no se correspondían y esto la llenó de sensaciones de angustia y malestar, mostrando retrocesos, a niveles 2 de la APES, manifestando quejas, como se aprecia en este párrafo: "[CVND) Si tuviera que utilizar emociones para ti- tular esta narración; utilizaría: la duda, la inseguridad, el desconcierto, y la ausencia de criterio" (2. Surgimiento. Marcador: Emociones dolorosas)].

En la narración \#10, coincidiendo con que la madre de Mónica tenía que hacerse una mamografía, explica que nota el cambio (nivel 6 de la APES) porque no siente tanto "dolor" por sí misma, pero la posibilidad de que le ocurra a alguien cercano la hace sentir lo mismo que siente por sus tumores. Se observa, entonces, cómo converge este tema con el de "evitar el sufrimiento a los demás", y cómo las emociones asociadas a "su vivencia de sentir la enfermedad como un cáncer" surgen de nuevo ante la posibilidad de que le ocurra a los demás. Así Mónica escribe: “[(VND) Es cierto que ya be dejado de llorar, incluso de sentir pena y tristeza, y sin embargo siento la preocupación porque vuelva a ocurrir, no a mí, sino en mi alrededor (5. Aplicación. Marcador: Bastante, pero no todavía resolviendo el problema)...]”.

Aquí podemos observar cómo converge este tema con el de "evitar el sufrimiento a los demás", y cómo las emociones asociadas a "su vivencia de sentir la enfermedad como un cáncer" surgen de nuevo ante la posibilidad de que le ocurra a los demás. Para Mónica esto sería un problema recurrente (marcador para el nivel 2). La comunidad de voces reconoce que una vozproblemática surge continuamente.

En la narración \#12 ocurre algo significativo que queremos destacar. Es también, una de las narraciones más extensas. De hecho, en ella llega a un 6.5, el nivel más alto en diversos momentos de todas las narraciones en todos los temas. Es en esta narración cuando Mónica reconoce que la solución pasa por no prestar atención al tema (correspondiente al nivel 6 de la APES). Concretamente Mónica escribió: "... simplemente he dejado de focalizar mi atención en mi pecho, mis tumores, y he dirigido el foco a otros elementos de mi alrededor; aspectos que me resultan gratificantes, motivadores y divertidos" (6.5. Solución del problema. Marcador: Notando el cambio)].

Este punto nos parece fundamental en la evolución y la resolución de esta experiencia problemática. Ésta se mantiene, desde ese momento, en niveles de Insight que reafirman la comprensión del problema con oscilaciones entre niveles 6 y 5 , pero con notables retrocesos. Podemos inferir que Mónica ha alcanzado niveles altos de asimilación de la experiencia problemática y se da cuenta del cambio que ha experimentado. Así Mónica escribió “... El El año pasado no pude realizar la travesía a nado, mi duelo me lo impidio, pero este año, (VD) sentí una emoción al tirarme al mar que jamás habia sentido, reconoci el placer y el gusto en una actividad, lo cual no valoraba con anterioridad a la experiencia (6.5. Solución del problema. Marcador: Notando el cambio)] [Y ahora redactando la narración me cuestiono sobre si reflexioné hace un par de horas el hecho de que se apreciara la falta de pecho con el bañador... y me sorprende pensar que el placer y la satisfacción fueron tales, que apenas me percaté sobre tal cuestión" (6.5. solución del problema. Marcador: Notando el cambio)]. 
○ Voces:

- VND : "Mónica la que siente miedo, incertidumbre, temor"

- VD: "Mónica la tranquila, sosegada, sin tensión"

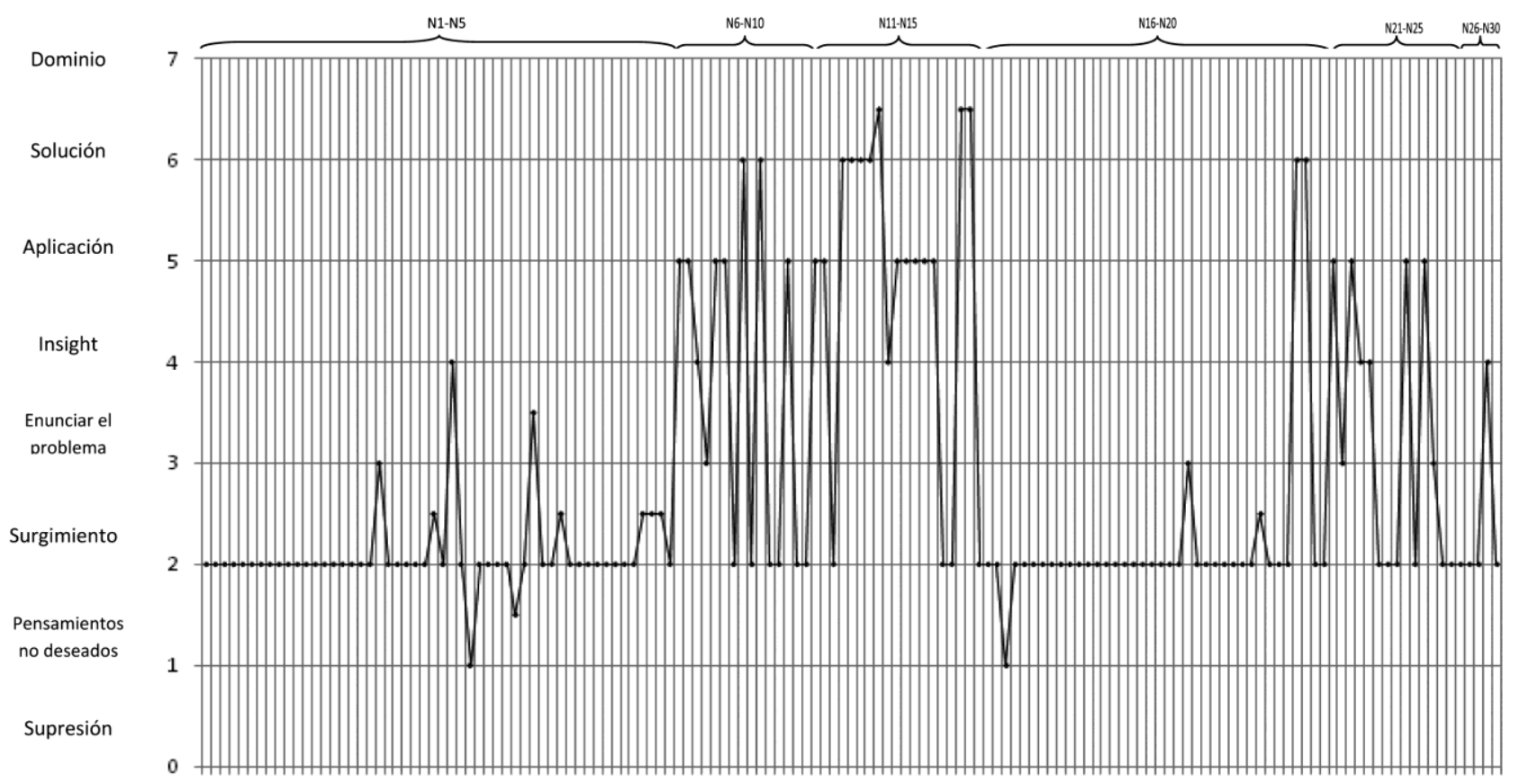

Figura 2. Proceso de asimilación del tema del temor, incertidumbre y miedos sobre el cáncer.

Voces:

- VND: "Mónica la que no sabe cómo afrontar el problema"

- VD: "Mónica la que sabe afrontar los problemas de su vida"

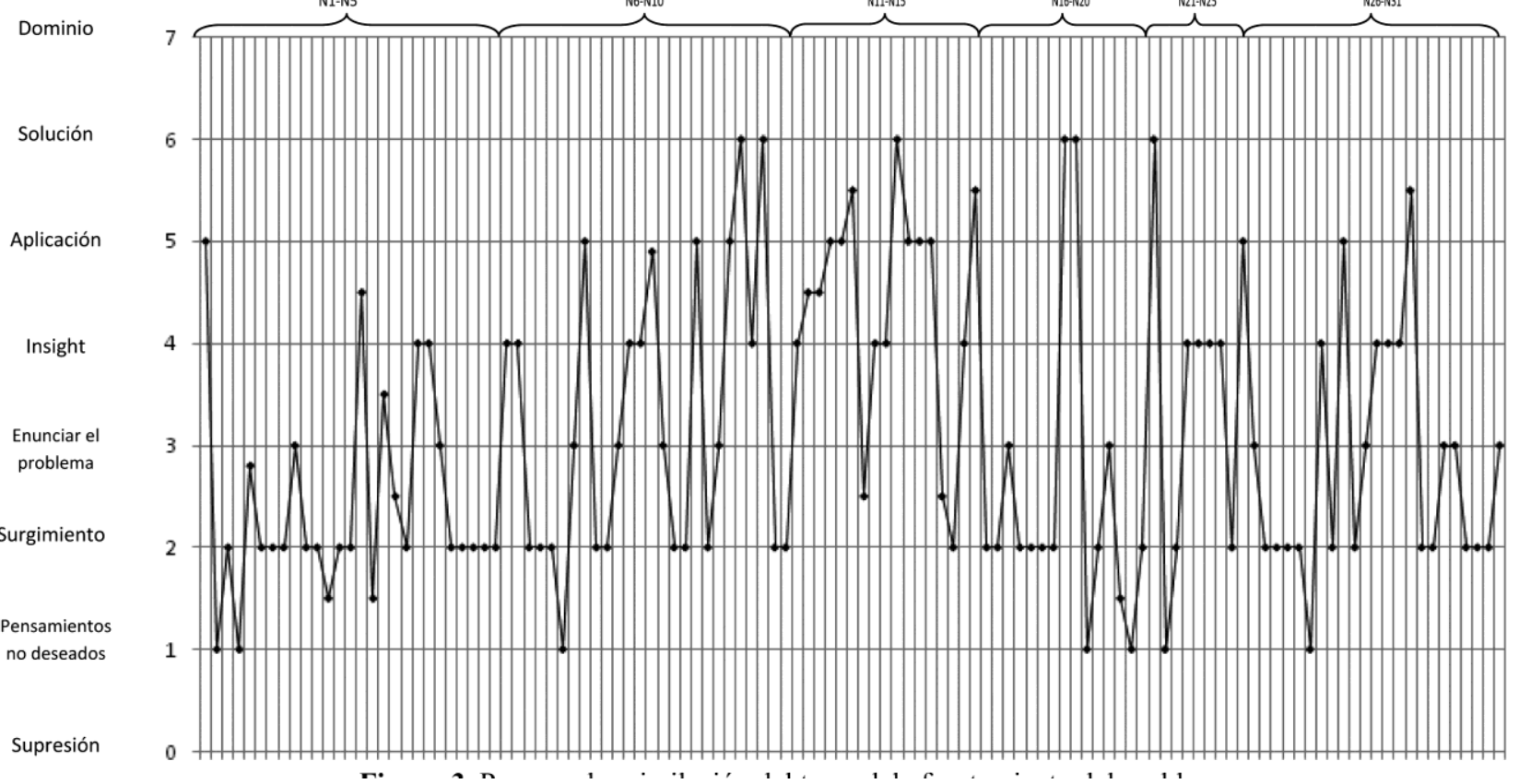

Figura 3. Proceso de asimilación del tema del afrontamiento del problema. 
Sin embargo, en la narración \#16, retrocede a niveles bajos de la APES. Esto coincide con el diagnóstico de cáncer de mama de una conocida de la familia. Aparecen, así una cadena importante de niveles 2. Por ejemplo, Mónica escribe: "[(VND) Mi mundo se desmorona, se desestabiliza y siento que tengo un miedo tan irracional que me asusta, (2. Surgimiento. Marcador: Sentirse vulnerable)] de nuevo vuelvo a experimentar un vacio tan inmenso que me aterra, y me paraliza" (2. Surgimiento. Marcador: Sentirse atrapado/bloqueado)].

La narración \#19 está escrita después de haber estado hospitalizada durante 4 días, en el hospital. Acudió por un fuerte dolor de pecho, y la dejaron en observación. Tras esta hospitalización, Mónica escribe, usando una canción, lo siguiente, alcanzando un nivel 6 de la APES. Veamos,

\section{De algo estoy segura \\ [(VD) ya no eres el fantasma \\ Que me rondaba haciendo un callejón \\ Cada segundo donde te pensaba (6. Solución del problema. Marcador: Notando el cambio) \\ (VD)Hoy no es pasión lo que siento \\ No es pena ni tormento \\ Hoy he salido de ti y entre lágrimas vi \\ Romperse el firmamento (6. Solución del problema. Marca- dor: Notando el cambio)]}

Inferimos, pues, que alcanza la asimilación de su experiencia problemática al darse cuenta del cambio en sus emociones respecto al miedo de tener un cáncer. Nos parece significativo lo que expresa en estos párrafos porque, de alguna manera, ante una situación que ella, en anteriores ocasiones, vivía con mucho miedo, tristeza e incertidumbre, ahora se da cuenta de que ya no la experimenta del mismo modo.

A partir de la narración \#21 retrocede a niveles de Insight y de clarificación (3 y 4, respectivamente), manteniéndose de este modo hasta la narración \#23 donde retrocede a niveles 2 de la APES cuando tiene que acompañar a su madre a hacerse una ecografía. Mónica escribe"... [(VND) Pasé miedo, nervios, me costaba tragar, notaba sequedad en la boca" (2. Surgimiento. Marcador: Emociones dolorosas)]. En la narración \# 28 coincidente con las vísperas de las fiestas navideñas Mónica muestra niveles 2 que se mantienen hasta el final. Por ejemplo, Mónica escribió “...A lo largo de los días asumi que llega la Navidad, los villancicos, los turrones ... [(VND) y sin ganas de celebrar ni de sonreír, sin contagiarme de eso que para mí resulta absurdo "espiritu de la Navidad", sigo sintiéndome igual o más triste que antes, (2. Surgimiento. Marcador: Emociones dolorosas)].... A lo largo de la semana, [(VND) siento un vacio, desánimo y apatia que se agudiza conforme a los días" (2. Surgimiento. Marcador: Emociones dolorosas)].

Tras esto aparece un pico con un nivel de Insight sobre el problema en la narración 30, donde Mónica asume que lo que debe hacer es aprender a convivir con el dolor. Final- mente termina en la última narración escribiendo este último párrafo:

[(VND)Pasan los días en soledad, una soledad que al principio era considerada como elegida, pero que dado el transcurso de la misma pasa a ser impuesta, auto impuesta que, en ocasiones la siento con vida propia vetando las ganas de llorar (2. Surgimiento. Marcador: Emociones dolorosas)], [(VND) en la que me siento asfixiada, ahogada (2. Surgimiento. Marcador: Emociones dolorosas)].

\section{Surgimiento de un nuevo marcador}

Es importante mencionar para seguir con la exposición de los resultados, la aparición de un posible nuevo marcador en el contexto narrativo. Tras analizar detenidamente las narraciones, nos dimos cuenta de que cuando Mónica alcanzaba el Insight, en ocasiones lo hacía gracias a reflexiones que se hacía ella misma sin ayuda de ningún otro externo. Esta forma de alcanzar el Insight, sin embargo, no se correspondía con ningún marcador de la APES descrito hasta el momento y lo denominamos, de mutuo acuerdo entre todas las jueces, como "reflexión elaborada". La definimos como: "Pregunta retórica que se hace la participante. Al no haber respuesta posible por parte de un yo externo, la forma interrogativa refuerza la afirmación obligando a detenerse a analizar y a comprender la cuestión. La participante llega al Insight con la respuesta implícita a la pregunta planteada."

Un ejemplo de este marcador sería: "En ocasiones anteriores he sentido tristeza, desesperanza y miedo... ¿me estaré acostumbrando a la situación? ¿Será que la he aceptado? o más bien ¿he aprendido a resignarme? (\#6. Código 4 APES; Tema: Temor, incertidumbre y miedos sobre el cáncer).

Consideramos importante este marcador por la frecuencia con la que aparece respecto a otros marcadores del nivel 4 (Insight). Así, en los párrafos analizados de las narraciones de Mónica, observamos que el marcador "juntar las piezas de forma distinta" apareció en 9 ocasiones de 34 Insights $(26.47 \%)$. Tras este marcador, aparece en 7 ocasiones (20.59\%) el marcador de "aceptación de hechos" (típico de estudios anteriores con la terapia lingüística de evaluación). $\mathrm{E}$, igualmente, en 7 ocasiones (20.59\%) el que proponemos en este trabajo la "reflexión elaborada".

\section{Beneficios para Mónica de las narraciones}

Con el fin de evaluar cómo había sido la tarea narrativa, le pedimos a Mónica, tras 15 narraciones escritas, que respondiera a una serie de preguntas. Fundamentalmente, queríamos que nos dijera cómo había vivido el proceso, qué había sido lo más útil y lo más difícil y cualquier comentario que quisiera añadir. Puesto que sus respuestas eran todas positivas y reconocía que le era útil escribir y que sentía bienestar al hacerlo, se decidió que siguiera escribiendo hasta llegar a unas 30 narraciones. Al terminar el estudio, respondió a una serie de preguntas (en enero de 2010) que exploraban la 
evolución y el impacto que tenía sobre ella el hecho de escribir sobre su experiencia.

En las preguntas respondidas a mediados del proceso explicó que la sensación de bienestar aumentaba al escribir y que conforme redactaba lo que le ocurría, su "tensión disminuía" e "incluso llegó a convertirse en un momento de desahogo y de despojar residuos de la experiencia". Comentó, además, que el hecho de escribir le ofrecía la oportunidad de tener una visión más detallada de su experiencia, de cómo reaccionaba y cómo experimentaba esa situación. También nos explicó que escribir sobre su experiencia le permitió tomar conciencia de cómo había ido evolucionando y que le proporcionaba bienestar el hecho de escribir sobre cómo afrontaba la situación y darse cuenta de que podía gestionar esa situación. A finales del proceso, comentó lo siguiente: "El hecho de plasmar las vivencias, no solo las respectivas a los tumores, sino aquellas que de forma directa o indirecta determinaban mi relación con el momento presente, ha significado una descarga".

También reconoce que el hecho de escribir le permitió tomar conciencia sobre cómo se sentía. Destacó, además lo siguiente: "Por primera vez he parado, he esperado, me he tomado tiempo, muchas veces impacientemente y he dejado fluir aquello que he reprimido en secreto durante mucho tiempo. Lo más importante ha sido compartir la experiencia, desvelar mis emociones y mis pensamientos a mi misma de forma explícita".

También queremos destacar el valor que le daba Mónica a las narraciones como se aprecia en lo siguiente: "Un aspecto muy significativo y que ha generado la sensación de confianza y por tanto la creación de un espacio seguro en el que poder sin ningún condicionante, expresar libremente mis emociones, ha favorecido la expresión y redacción no sólo de mi vivencia con los tumores, sino al contexto que fragua, crea y en ocasiones alienta mi momento presente."

\section{Discusión}

El modelo de asimilación ha crecido principalmente gracias a los estudios de caso (Honos-Webb et al., 1998; Knobloch et al., 2001; Stiles, 2002; Stiles et al., 1990, 1991, 1992; Varvin \& Stiles, 1999). El desarrollo gradual del modelo ilustra cómo las observaciones de caso han impregnado el modelo, refinándolo, elaborándolo, y clarificándolo (Stiles, 2003). Desde este punto de vista, es interesante reflexionar, en primer lugar, sobre las ventajas de un estudio de asimilación a través de narraciones.

Se ha señalado la cercanía del modelo de asimilación con la perspectiva narrativa (Osatuke et al., 2004; Stiles, HonosWebb \& Lani, 1999). Con este trabajo se puede asumir la posibilidad de analizar mediante el modelo de asimilación narraciones en lugar de sesiones de terapia (centro de los estudios en el modelo de asimilación, véase, Stiles, 2002). Tras analizar los datos de Mónica consideramos que las narraciones nos dan la posibilidad de conocer cómo le afecta el diagnóstico viéndose truncada su identidad y su forma de entender el mundo en general, tal y como planteó Frank (1995).
De ahí que, en el caso de Mónica, el diagnóstico y toda la problemática que de éste se desprende rompe con la coherencia que tiene de su vida, algo que se aprecia en sus escritos. La aparición de un fibroadenoma y de los tumores continuos provoca en Mónica que su vida se desequilibre por este hecho y que todo su mundo, desde ese momento, gire en torno a la enfermedad. Se trata, pues, de una oportunidad, si queremos considerarlo en términos positivos, para replantearse su historia personal, su diálogo para con ella misma y su reformulación de lo que le ocurría, con el fin de enriquecer el self, o su identidad y hacerla válida para el afrontamiento de la enfermedad. En este sentido se pronuncian autores como Brody (1987), Frank (1995), Bhuvaneswar \& Shafer (2004) y Neimeyer (1994), en el contexto de la enfermedad, sobre todo del cáncer.

En la línea defendida por diversos autores (Neimeyer, 1994; Polkinghorne, 1988; Sarbin, 1986; Spence, 1982), podemos asumir que escribir narraciones ha sido útil a Mónica, para organizar, dar sentido y coherencia a la experiencia así como un medio para elaborar sus vivencias. Este uso funcional de las narraciones, está relacionado con los beneficios psicológicos de ellas.

Como recogen Kerner \& Fitzpatrick (2007), la escritura puede utilizarse como un método para disminuir la inhibición emocional, animar la revelación y la expresión emocional. Esta idea entronca con la voz de Mónica que le decía que "no debía quejarse" y "no debía hacer sufrir a los demás". De hecho, estas voces experimentan cambios a lo largo de las narraciones. Vemos, en sus escritos, cómo disminuye el peso de la voz dominante. Puesto que estas voces hacían que Mónica reprimiera su necesidad de expresar lo que le podría ocurrir, consideramos que en la narración se sentía libre de decir qué estaba sintiendo, pensando y haciendo, sin límites. Así, la escritura nos permite manifestar y elaborar sentimientos sin el riesgo que puede suponer de aislamiento social al verbalizar de forma continua ansiedad o malestar (Pennebaker, 1997).

Con este estudio hemos visto cómo se puede explicar la asimilación de una experiencia problemática a través del modelo y junto con la APES. El modelo propone (véase Honos-Webb \& Stiles, 1998) un diálogo entre las voces que nos resulta adecuado para entender cómo cambian las experiencias problemáticas y cómo se relacionan esas voces hasta llegar a estar integradas en el self, y esto se hace evidente, igualmente, en las narraciones.

Dos cuestiones fundamentales sobre las que nos debemos centrar se refieren, en primer lugar, a si hubo o no hubo asimilación en Mónica, y, en segundo lugar, al tipo de patrón de asimilación en todas sus experiencias problemáticas. Como expusimos en el apartado de Resultados, la mayoría de las experiencias problemáticas de Mónica analizadas en este estudio llegan a asimilarse en diversos momentos. Sólo en la experiencia de "ordenar el exterior para ordenar el interior" se queda en niveles bajos.

Respecto a la asimilación y siguiendo la perspectiva teórica sobre las voces asumida en el modelo de asimilación 
(Honos-Webb \& Stiles, 1998), podríamos inferir que Mónica reconoce que la voz. dominante no puede llevarse siempre a la práctica porque en su vida van surgiendo situaciones novedosas que tiene que ir asimilándolas, adaptando sus voces a las circunstancias. Cabe señalar, además, que la situación vivida por Mónica se podría entender como una experiencia vital amenazante. Así, este tipo de experiencias van a ser más difíciles de asimilar.

Podemos inferir que mediante sus narraciones Mónica se dio cuenta de la importancia que para ella tenía sentirse tranquila ante la situación de vivir el diagnóstico como si fuera un posible cáncer, mantener una estabilidad a nivel emocional, así como sentir orden en su vida, saber afrontar el problema, quejarse por su sufrimiento, y no sentirse culpable si no visitaba a sus familiares enfermos o si hacía sufrir a los demás. Inferimos que Mónica asimila que no puede controlar todos los aspectos de su vida, ni sus emociones. Reconoce que esas exigencias que ella misma se plantea, en momentos de crisis no le ayudan, sino que le producen malestar y sufrimiento. Es decir, deducimos que, según los procesos de asimilación encontrados, aprende a vivir con todo ello, asimilándolo en su self. Éste se enriquece de esta manera en diversos momentos de su proceso, ya que aunque sigue queriendo controlar ciertos aspectos de su vida, y mantener un orden y un equilibrio reconoce que esto no puede ser siempre así.

Además, podemos asumir que la APES es sensible al cambio y a la evolución de la participante. Respecto al cambio los datos sugieren distintos niveles de análisis. Mónica comenta el beneficio de usar las narraciones y se siente motivada a hacerlo por dichos beneficios, hasta el final del estudio. Además se produce asimilación en siete de sus ocho experiencias problemáticas, aunque con retrocesos, como luego comentaremos. Si observamos las puntuaciones en el pretest y el postest vemos que hay cambios en la mayoría de las subescalas donde presentaba puntuaciones altas en el pretest (en el SCL-90-R), aunque sigue estando en intervalos elevados o por encima del punto de corte en las subescalas del HAD y del Mini-Mac. Podemos inferir que estos datos sugieren que la APES es una medida sensible al proceso de cambio (Caro Gabalda, 2006a,b, 2007, 2008; Detert et al., 2006; Osatuke, Humphreys et al., 2005; Stiles, 2002; Stiles et al., 1990, 1991) y sensible al estado psicológico de nuestra participante que tiene que intentar asimilar una experiencia que podemos calificar de amenazante para ella y lo intenta hacer mediante narraciones.

Como se ha visto, aparecen notables retrocesos tanto en los patrones de asimilación de prácticamente todas las experiencias problemáticas. No obstante, como señalan Caro Gabalda y Stiles (2009) que aparezcan retrocesos no parece ser un impedimento para obtener buenos resultados terapéuticos en la comparación estándar pretest-postest. Ahora bien, éste es un resultado interesante en el contexto de asimilación que se señala en diversos trabajos.

Los estudios de caso sobre asimilación demuestran que el progreso de la secuencia de APES no siempre es continuo y regular (Caro Gabalda \& Stiles, 2009; Caro Gabalda \& Stiles, remitido para su publicación) como hemos podido apreciar en las experiencias de Mónica analizadas aquí. Este patrón de asimilación de las experiencias analizadas de Mónica reproduce otros estudios que se han realizado con el modelo (Detert et al., 2006; Goodridge \& Hardy, 2009; Honos-Webb et al., 1998; Osatuke, Glick et al., 2005). Tras diversos estudios se ha llegado a la conclusión de que existen diferencias importantes respecto al patrón de asimilación entre distintos abordajes terapéuticos, como ya se ha señalado en la Introducción.

En este sentido, los análisis realizados con los datos de Mónica coinciden, o se acercan más a los resultados sobre el proceso de asimilación en terapias cognitivas. Por tanto, hemos encontrado un resultado interesante a confirmar en estudios sucesivos. Sin terapia y con un enfoque narrativo también existen retrocesos y "saltos" (patrón de sierra dentada) en el proceso de asimilación de experiencias problemáticas (al menos en el estudiado en el presente trabajo). Las principales explicaciones esbozadas en la Introducción se encuadraban, desde la perspectiva de la multiplicidad de voces, en torno a dos principales, la zona de desarrollo próximo y la bipótesis de la balanza. Estas dos explicaciones principales (Caro Gabalda \& Stiles, 2009; Caro Gabalda \& Stiles, remitido para su publicación) se han planteado en el contexto terapéutico y destacan la relación paciente-terapeuta y la sintonía (o no) entre ellos. Ahora bien, en este contexto narrativo debemos abrirnos a la posibilidad de otras explicaciones.

Podríamos asumir, de forma tentativa, a la espera de un estudio específico para ello, que la asimilación de las experiencias problemáticas de Mónica no se ha mantenido debido a distintas cuestiones. En primer lugar, podría deberse a que no hay un refuerzo externo (como podría ser un terapeuta) que reconozca ese cambio y lo refuerce de manera que esos cambios se consideren útiles como recursos y cambios positivos. Igualmente, podríamos asumir que, quizás, se haya dejado de trabajar con narraciones demasiado pronto y Mónica necesitara más tiempo para asimilar las nuevas experiencias que aparecían en su vida. Honos-Webb, Stiles, Greenberg y Goldman (1998) señalan a esta explicación como una de las posibles en el contexto de sesiones de terapia. Finalmente, cabe inferir, además, que esta situación la podríamos entender como una experiencia vital amenazante. En el contexto del modelo de asimilación se asume que no todas las experiencias son igualmente asimilables y que experiencias problemáticas amenazantes puede que sean más difíciles de asimilar (Varvin \& Stiles, 1999). Teniendo en cuenta que Mónica durante todo el proceso de escribir sus narraciones seguía sometida a controles médicos rutinarios en el IVO, y personas cercanas a ella eran diagnosticadas con cáncer, la experiencia problemática, aunque se hayan dado cambios en ella, no ha dejado de estar presente.

Un dato que nos parece relevante señalar sería la aparición de un marcador nuevo en la APES, en este contexto narrativo. Tradicionalmente, los marcadores nos sirven como guía y criterio (serían "pistas" sobre cada nivel APES) 
para ofrecer los códigos APES, aunque hasta ahora sólo se disponía de ellos en un contexto terapéutico (Honos-Webb, Stiles \& Greenberg, 2003; Honos-Webb, Stiles, Greenberg \& Goldman, 1998).

En este sentido, en el nivel de Insight, Mónica hace en varias ocasiones una "reflexión elaborada", tal y como hemos denominado, tentativamente, a este nuevo marcador. Hemos analizado las narraciones de Mónica sabiendo que se trata de un contexto en el que ella sola escribe sin refuerzo ni apoyo de nadie más. En este sentido, los marcadores de la APES están formulados en el contexto de una terapia - usual, en la que existe un terapeuta que plantea una serie de cuestiones y se discuten ciertas experiencias problemáticas. Como en el caso de Mónica no se da esta situación, ella misma se plantea ciertas reflexiones que va elaborando sola. Es decir, hemos visto que cuando Mónica llegaba al Insight, reconocíamos en algunos momentos que comprendía la experiencia problemática a través de reflexiones elaboradas. Esta manera de comprenderla sugiere este nuevo marcador. Por tanto, sería conveniente en futuros trabajos, similares al presente, investigar si este marcador se ve en más narraciones, e, igualmente, en análisis de sesiones de terapia, contribuyendo a la ampliación del modelo de asimilación en general, y de la escala APES en particular.

Recientemente se ha relacionado el modelo de asimilación con las teorías metacognitivas (Osatuke, Stiles, Barkham, Hardy \& Shapiro, en prensa). Creemos que esto es especialmente evidente en el nivel 4 o de Insight con sus diversos marcadores. El "nuevo" marcador esbozado por nosotras, "reflexión elaborada", sería un buen ejemplo de esta capacidad metacognitiva. Así, este "nuevo" marcador se puede relacionar con teorías que entienden la narración del sí mismo como metáfora que implica un sí mismo dialogal que utiliza el autohabla y presupone una relación dialogal entre el Yo y el Mí (Hermans, 1996; Hermans-Jansen, 1995). Nuestro sí mismo es parte de una narrativa (Bruner, 1986, 1990, 2004; Howard, 1991). Los seres humanos damos sentido a lo que nos sucede mediante historias que necesitan tener sentido y ser coherentes para que sean viables y funcionales (Adler, Skalina \& McAdams, 2008). Podemos apreciar este intento de dar sentido en diversos momentos de las narraciones de Mónica. Y sobre todo en los momentos en dónde apareció recogido este "nuevo" marcador.

Como hemos comentado, reconociendo que en la narración no existe "otro" externo que pueda dialogar con ella y rebatirle ciertas ideas o trabajarlas con el fin de llegar a un objetivo, esa reflexión planteada por la comunidad de voces proporciona una vía para ese diálogo entre ellas. Éste lleva a una mayor comprensión e integración de las voces opuestas. En este sentido, es importante entender que este posible nuevo marcador puede darse en estas circunstancias en las que las voces dominantes y no dominantes hablan entre ellas y se plantean las distintas vías por las que llegar a integrarse.

En este sentido Mónica demuestra que es capaz de reconocer su experiencia subjetiva, identificando pensamientos y emociones, así como reflexionando sobre ellos (Dimaggio,
Hermans \& Lysaker, 2010). Este marcador muestra cómo Mónica fue capaz de establecer un diálogo saludable (una metaposición o una integración metacognitiva; Dimaggio, Salvatore, Azzara \& Catania, 2003) entre las voces, que facilitó el establecimiento de puentes de significado, fundamentalmente, en el nivel de Insight (APES 4) que es el nivel dónde se aprecia mejor el diálogo y la comprensión entre voces (Stiles \& Glick Brinegar, 2007).

\section{Ventajas y limitaciones de este estudio}

La perspectiva narrativa nos ha facilitado entender a Mónica conociendo su problema de forma detallada, atendiendo a los matices de su experiencia problemática, viendo momento a momento lo que le ocurre, fijándonos en cada palabra, en cada evolución de sus narraciones. En definitiva, hemos hecho una primera aproximación al tema de la vivencia de una paciente en un servicio oncológico que describe cómo se experimenta la amenaza de poder tener un cáncer y cómo se articula la experiencia a través de las narraciones y del modelo de asimilación.

En esta línea, reconocemos que cuando trabajamos con narraciones, un asunto clave es el nivel de inferencia que hacemos de los procesos de los participantes. Con ello queremos decir que asumimos que la confianza en los resultados puede verse mermada. Sin embargo la utilización de jueces en el análisis de los datos se hace con el fin de disminuir esas inferencias, puesto que se trata de llegar a un acuerdo sobre lo que ha analizado.

Además, reconocemos la controversia que suponen los resultados de investigaciones cualitativas. Sin embargo, para dotar a este estudio de mayor rigor empleamos jueces con el fin de llegar a un acuerdo sobre los datos, de forma, que los datos fueran la consecuencia de un consenso y acuerdo. Además, para contribuir a su validez, los investigadores cualitativos ofrecen extractos de ellos para que sean confirmados por la comunidad interpretativa (Stiles, 1993). Eso es lo que hemos hecho aquí, ofreciendo, aunque brevemente, algunos de estos extractos de las narraciones de Mónica. Ofrecer los pasajes seleccionados, junto con la asignación del nivel APES y el marcador, tiene el objetivo de que el lector juzgue y valore la evaluación que se ha hecho del dato. Con este acceso a los datos se puede comprobar cómo y por qué se ha realizado tal análisis.

Podríamos inferir, en función de nuestros datos, la necesidad de realizar investigaciones clínico -empíricas, utilizando estudios de caso con metodología como el modelo de asimilación que ha demostrado su utilidad para analizar el proceso del cambio.

Así, en una perspectiva cualitativa y dado que se analizan muchos enunciados, esto incrementa la confianza en nuestra teoría, lo que lo hace comparable a la confianza que podemos obtener de un estudio encaminado a la comprobación de hipótesis (Stiles, 2007). Unos pocos casos, analizados sistemáticamente, que coinciden con una teoría de una forma muy precisa aumentan nuestra confianza en la teoría. La se- 
rie de estudios de caso realizados sobre el modelo de asimilación, a los cuales cabe añadir el aquí presentado, nos permiten asumir y defender el modelo de asimilación y sus propuestas, así como las ventajas del estudio de casos.

Para finalizar, el presente trabajo deja una puerta abierta a la investigación siguiendo la línea planteada en estas pági-

\section{Referencias}

Adler, J. M., Skalina, L. M. \& McAdams, D. P. (2008). The narrative reconstruction of psychotherapy and psychological health. Psychotherapy Research, 18, 719-734. doi :10.1080/10503300802326020

Bajtin (1984). Problems of Dostoevsky's poetics. Minneapolis: University of Minnesota Press. (Trabajo original publicado en 1929).

Bell, S. (2002). Photo images: Jo Spence's narratives of living with illness. Health: An Interdisciplinary Journal for the Social Study of Health, Illness and Medicine, 6, 5-30. doi:10.1177/136345930200600102

Bhuvaneswar, C., \& Shafer, A., (2004). Survivor of that time, that place: clinical uses of violence survivors' narratives. Journal of Medical Humanities, 25, 109-127. doi: 10.1023/B:JOMH.0000023175.21955.dc

Brody, H. (1987). Stories of sickness. New Haven, CT: Yale University Press.

Bruner, J. (1986). Actual minds, possible worlds. MA: Harvard University Press.

Bruner, J. (1990). Acts of meaning. Cambridge, MA: Harvard University Press.

Bruner, J. (2004). Life as narrative. Social Research, 71, 691-710.

Caro Gabalda, I. (2006a). The assimilation of problematic experiences in linguistic therapy of evaluation: How did María assimilate the experience of dizziness? Psychotherapy Research, 16, 422-435. doi:10.1080/10503300600756436

Caro Gabalda, I. (2006b). The assimilation of problematic experiences in the context of a therapeutic failure. Psychotherapy Research, 16, 436-452. doi: $10.1080 / 10503300600743897$

Caro Gabalda, I. (2007). La asimilación de experiencias problemáticas en la terapia lingüística de evaluación. ¿Cómo asimiló María su incapacidad para hacer cosas? [The assimilation of problematic experiences in linguistic therapy of evaluation: How did María assimilated her inability to do things?] Boletín de Psicología, 89, 47-73.

Caro Gabalda, I. (2008). Assimilation of problematic experiences in Linguistic Therapy of Evaluation: a case study. Journal of Constructivist Psychology, 21, 151-172. doi: 10.1080/10720530701853735

Caro Gabalda, I. \& Ibáñez Guerra E. (1992). La Escala Hospitalaria de Ansiedad y Depresión. [The Hospital Anxiety and Depression Scale] Boletín de Psicología, 36, 43-69.

Caro Gabalda, I., \& Stiles, W.B. (2009). Retrocesos no contexto de terapia linguística de avaliaçâo. Análise Psicologica, 2, 199-212.

Caro Gabalda, I. \& Stiles, W.B. (2012). Irregular assimilation progress: Reasons for setbacks in the context of linguistic therapy of evaluation. Psychother Res, 23(1), 35-53. doi: 10.1080/10503307.2012.721938.

Derogatis, L. R. (2002). SCL-90-R. Cuestionario de 90 Sintomas. [SCL-90-R. 90 Symptons Questionnaire]. Madrid: TEA.

Detert, N. B., Llewelyn, S., Hardy, G.E., Barkham, M., \& Stiles, W. B. (2006). Assimilation in good-and poor-outcome cases of very brief psychotherapy for mild depression: An initial comparison. Psychotherapy Research, 16, 393-407. doi: 10.1080/ 10503300500294728

Dimaggio, G., Salvatore, G., Azzara, C. \& Catania, D. (2003). Rewriting selfnarratives: The therapeutic process. Journal of Constructivist Psychology, 16, 155-181. doi: 10.1080/10720530390117920

Dimaggio, G., \& Stiles, W.B. (2007). Psychotherapy in light of internal multiplicity. Journal of Clinical Psychology, 63, 119-127. doi: 10.1002/jclp.20335

Dimaggio, G., Hermans, H. J. M., \& Lysaker, P. H. (2010). Health and adaptation in a multiple self. Theory and Psychology, 20, 379-399. doi:10.1177/0959354310363319

Elliot, R. (1985). Helpful and nonhelpful events in brief counseling interviews: An empirical taxonomy. Journal of Counseling Psychology, 32, 307322. doi: 10.1037/0022-0167.32.3.307

Fisher, W. (1985). The narrative paradigm: In the beginning. Journal of Communication, 35, 74-89. doi: 10.1111/j.1460-2466.1985.tb02974.x nas así como el estudio del ser humano desde el modelo de asimilación y desde la perspectiva narrativa como un enfoque interesante y detallado sobre el conocimiento y la experiencia humana.

Frank, A. W. (1992). The Pedagogy of suffering: moral dimensions of psychological therapy and research with the ill. Theory and Psychology, 2, 467485. doi:10.1177/0959354392024004

Frank, A. W. (1994). Reclaiming an orphan genre. Literature and Medicine, 13, 1-21. doi : 10.1353/lm.2011.0180

Frank, A. W. (1995). The wounded storyteller: Body Illness, and Ethics. Chicago: The University of Chicago Press.

Frank, A. W. (1998). Just listening: narrative and deep illness. Family, Systems \& Health, 16, 197-212. doi: 10.1037/h0089849

Frank, A. W. (2000). Illness and autobiographical work: dialogue as narrative destabilization. Qualitative Sociology, 23, 135-156. Doi: 10.1023/A:1005411818318

Greenberg, M. A., Wortman, C. B. \& Stone, A. A. (1996). Emotional Expression and Physical Health: Revising Traumatic Memories or Fostering Self-Regulation? Journal of Personality and Social Psychology, 71, 588-602. doi: 10.1037/0022-3514.71.3.588

Goodridge, D., \& Hardy, G. E. (2009). Patterns of change in psychotherapy: An investigation of sudden gains in cognitive therapy using the assimilation model. Psychotherapy Research, 19, 114-123. doi: 10.1080/10503300802545611

Hardy, B. (1968). Towards a poetics of fiction: an approach through narrative. En M. Meek, A. Warlow, \& G. Barton, (Eds.), The Cool Web: the pattern of children's Reading (pp.12-23).Nueva York: Atheneum.

Henry, H., Stiles, W., \& Biran, M. (2005). Loss and mourning in immigration: Using the assimilation model to assess continuing bonds with native culture. Counseling Psychology Quarterly, 18, 109-119. Doi: 10.1080/09515070500136819

Hermans, H.J.M. (1996). Voicing the self: From information processing to dialogical exchange. Psychological Bulletin, 119, 31-50. doi: 10.1037/00332909.119.1.31

Hermans, H. J. M., \& Dimaggio, G. (Eds.). (2004). The dialogical self in psychotherapy: An introduction. Nueva York: Brunner-Routledge.

Hermans, H. J. M., \& Hermans-Jansen, E. (1995). Self-narratives: the construction of meaning in psychotherapy. Nueva York: Guilford Press.

Hermans, H. J. M., Kempen, H. J. G., \& van Loon, R. P. P. (1992). The dialogical self: Beyond individualism and rationalism. American Psychologist, 47, 23-33. doi: 10.1037/0003-066X.47.1.23

Hewitt, J. P. (1989). Self and society: a symbolic interactionist perspective (6th Ed.). Needham Heights, MA: Allyn and Bacon.

Honos-Webb, L., Surko, M., \& Stiles, W. B. (1998). Manual for rating assimilation in psychotherapy. Manuscrito sin publicar. Miami University, Ohio.

Honos-Webb, L., Surko, M., Stiles, W. B., \& Greenberg, L. S. (1999). Assimilation of voices in psychotherapy: The case of Jan. Journal of Counseling Psychology, 46, 448-460. doi: 10.1037/0022-0167.46.4.448

Honos-Webb, L., \& Stiles, W. B. (1998). Reformulation of assimilation analysis in terms of voices. Psychotherapy, 35, 23-33. doi: 10.1037/h0087682

Honos-Webb, L., Stiles, W. B., Greenberg, L. S., \& Goldman, R. (1998). Assimilation analysis of process-experiential psychotherapy: A comparison of two cases. Psychotherapy Research, 8, 264-286. doi: 10.1080/10503309812331332387

Honos-Webb, L., Stiles, W. B., \& Greenberg, L. S. (2003). A method of rating assimilation in psychotherapy based on markers of change. Journal of Counseling Psychology, 50, 189-198. doi:10.1037/0022-0167.50.2.189

Howard, (1991). Culture tales: A narrative approach to thinking, crosscultural psychology, and psychotherapy. American Psychologist, 46, $187-$ 197. doi: 10.1037/0003-066X.46.3.187

Kerner, E. A., \& Fitzpatrick M. R. (2007). Integrating writing into psychotherapy practice: a matrix of change processes and structural dimen- 
sions. Psychotherapy: Theory, Research, Practice, Training, 44, 333-346. doi: 10.1037/0033-3204.44.3.333

Knobloch, L. M. Endres, L. M., Stiles, W. B., \& Silberschatz, G. (2001). Convergence and divergence of themes in successful psychotherapy: An assimilation analysis. Psychotherapy, 38, 31-39. doi: 10.1037/00333204.38.1.31

Lani, J. A., Glick, M. J., Osatuke, K., Brandenburg, C., Gray, M. A., Humphreys, C. M., Reynolds, D., \& Stiles, W. B. (2002). Markers of assimilation manual. Manuscrito sin publicar. Miami University, Ohio.

Leiman, M., \& Stiles, W. B. (2001). Dialogical sequence analysis and the zone of proximal development as conceptual enhancements to the assimilation model: The case of Jan revisited. Psychotherapy Research, 11, 311-330. doi :10.1080/713663986

Lepore S. (1997). Expressive writing moderates the relation between intrusive thoughts and depressive symptoms. Journal of Personality and Social Psychology, 73, 1030-1037. doi: 10.1037/0022-3514.73.5.1030

López-Roig, S., Terol, M. C., Pastor, M. A., Neipp, M. C., Massutí, B., Rodríguez-Marín, J., Leyda, J. I., Martín-Aragón, M. \& Sitges, E. (2000). Ansiedad y depresión. Validación de la escala HAD en pacientes oncológicos. [Anxiety and depression. Validation of HAD with oncological patients]. Revista de Psicología de la Salud, 12, 127-155.

Lumley, M. A., \& Provenzano, K. M. (2003). Stress management through written emotional disclosure improves academic performance among college students with physical symptoms. Journal of Educational Psychology, 95, 641-649. doi: 10.1037/0022-0663.95.3.641

Neimeyer, R. A. (1994). The role of client-generated narratives in psychotherapy. Journal of Constructivist Psychology, 7, 229-242. doi :10.1080/10720539408405232

Osatuke, K, Glick M. J., Gray, M. A., Reynolds, D. J. Jr., Humphreys, C. L., Salvi, L. M., \& Stiles, W.B. (2004). Assimilation and narrative: stories as meaning bridges. En L. Angus \& J. McLeod (Eds.), Handbook of narrative and psychotherapy: Practice, theory, and research (pp. 193-210). Thousand Oaks, CA: Sage.

Osatuke, K., Glick, M.J., Stiles, W.B., Greenberg, L.S., Shapiro, D.A., \& Barkham, M. (2005). Temporal patterns of improvement in clientcentered therapy and cognitive-behaviour therapy. Counselling Psychology Quarterly, 18, 95-108. doi: 10.1080/ 09515070500136900

Osatuke, K., Humphreys, C, Glick, M., Graff-Reed, R., McKenzie Mack, L., \& Stiles, W. B. (2005). Vocal manifestations of internal multiplicity: Mary's voices. Psychology and Psychotherapy: Theory, Research and Practice, 78, 21-44. doi: 10.1348/47608304X22364

Osatuke, K., Stiles, W.B., Barkhamn, M., Hardy, G., \& Shapiro, D. A. (en prensa). Relationships between mental states in depression: The assimilation model. Psychiatry Research.

Piaget, J. (1962). Play, dreams and imitation in childhood. Nueva York: Norton.

Piaget, J. (1970). Piaget's theory. En P. H. Mussen (comp.), Carmichael's manual of child psychology. ( $3^{\text {rd }}$ ed.) (pp. 703- 732). Nueva York: Wiley.

Pennebaker, J., Kiecolt-Glaser, J. \& Glaser, R. (1988). Disclosure of traumas and inmune function: health implications for psychoterapy. Journal of Consulting and Clinical Psychology, 56, 638-639.

Pennebaker, J. W., Colder, M., \& Sharp, L. K. (1990). Accelerating the coping process. Journal of Personality and Social Psychology, 58, 528-537. doi:10.1037/0022-3514.58.3.528

Pennebaker, J. W. (1997). Writing about emotional experiences as a therapeutic process. Psychological Science, 8, 162-166. doi:10.1111/j.14679280.1997.tb00403.x

Pennebaker, J. W., Mayne, T. J., \& Francis, M. E., (1997). Linguistic predictors of adaptative bereavement. Journal of Personality and Social Psychology, 72, 863- 871. doi: 10.1037/0022-3514.72.4.863

Polkinghorne, D. E. (1988). Narrative knowing and the human sciences. Albany: State University of New York Press.

Ricoeur, P. (1979). Introducción. [Introduction]. A. A. V. V. El tiempo y las filosofias (pp.11-35) Salamanca: Sígueme.
Ricoeur, P. (1997). Autobiografía intelectual [Intelectual self-biography]. Buenos Aires: Nueva Visión.

Rogers, C. (1959). A theory of therapy, personality and interpersonal relationships as developed by the client-centered framework. En S. Koch (Ed.), Psychology: A study of science. Vol. III. Formulations of the person and the social context (pp. 184-256). Nueva York: McGraw-Hill.

Sarbin, T. R. (1986). The narrative as root metaphor for psychology. En T. R. Sarbin (Ed.), Narrative psychology: The storied nature of human conduct (pp. 3-21). Nueva York: Praeger.

Schielke, H. J., Fishman, J.L, Osatuke K., \& Stiles W. B. (2009). Creative consensus on interpretations of qualitative data: The Ward Method. Psychotherapy, 19, 558-565.

Spence, D. P. (1982). Narrative truth and historical truth: memory and interpretation in psychoanalysis. Nueva York: W.W. Norton and Company.

Stiles, W. B. (1993). Quality control in qualitative research. Clinical Psychology Review, 13, 593-618.

Stiles, W. B. (2002). Assimilation of problematic experiences. En J. C. Norcross (Ed.), Psychotherapy relationships that work: Therapist contributions and responsiveness to patients (pp. 357-365). Nueva York: Oxford University Press.

Stiles, W. B. (2003). When is a case study scientific research? Psychotherapy Bulletin, 38, 6-11.

Stiles, W. B. (2005). Extending the Assimilation of Problematic Experiences Scale: Commentary on the Special Issue. Counselling Psychology Quarterly, 18, 85-93. doi: 10.1080/09515070500136868

Stiles, W. B. (2007). Theory-building case studies of counselling and psychotherapy. Counseling and Psychotherapy Research. 7, 122-127. doi: 10.1080/ 14733140701356742

Stiles, W. B. (2011). Coming to terms. Psychotherapy Research, 21, 367-384. doi: 10.1080/ 10503307.2011.582186.

Stiles, W. B., Elliott R., Llewelyn S., Firth-Cozens J., Margison F. R., Shapiro D. A., \& Hardy, G. (1990). Assimilation of problematic experiences by clients in psychotherapy. Psychotherapy, 27, 411-420. doi: 10.1037/00333204.27.3.411

Stiles \& Glick Brinegar, (2007). Insight as a stage of assimilation: A theoretical perspective. En L.G. Castonguay y C.E. Hill (Eds.), Insight in psychotherapy (pp. 101-118). Washington: APA.

Stiles, W. B., Honos-Webb, L., \& Lani, J. A. (1999). Some functions of narrative in the assimilation of problematic experiences. Journal of Clinical Psychology, 55, 1213-1226. doi: 10.1002/(SICI)10974679(199910)55:10<1213::AID-JCLP4>3.0.CO;2-1

Stiles, W. B., Meshot, C. M., Anderson, T. M., \& Sloan, W. W. (1992). Assimilation of problematic experiences: The case of John Jones. Psychotherapy Research, 2, 81-101. doi :10.1080/10503309212331332874

Stiles, W. B., Morrison, L. A., Haw, S. K., Harper, H., Shapiro, D. A., \& Firth-Cozens, J. (1991). Longitudinal study of assimilation in exploratory psychotherapy. Psychotherapy, 28, 195-206. doi: 10.1037/00333204.28.2.195

Varvin, S., \& Stiles, W. B. (1999). Emergence of severe traumatic experiences: An assimilation analysis of psychoanalytic therapy with a political refugee. Psychotherapy Research, 9, 381-404. doi :10.1080/10503309912331332821

Vogel, D. (1995). Perspectivas narrativas en la teoría y en la práctica. [Narrative perspectivas in theory and practice]. Revista de Psicoterapia, 6, 21-38.

Watson, M., Law, M., Dos Santos, M., Greer, S., Baruch, J., \& Bliss, J. (1994). The Mini-Mac: Further development of the Mental Adjustment to Cancer Scale. Journal Psychosocial Oncology, 12, 33-46. doi: 10.1300/J077V12N03_03

Zigmond, A. S., \& Snaith, R. P. (1983). The Hospital Anxiety and Depression Scale. Acta Psychiatrica Scandinavica, 67, 361 -370. doi: 10.1111/j.1600-0447.1983.tb09716.x

(Artículo recibido: 04-06-2012; revisado: 07-11-2012: aceptado: 07-11-2012) 